\title{
Du Journal de Mihail Sebastian au Retour du hooligan: une vie de Norman Manea
}

L'évasion dans la république des lettres

From Mihail Sebastian's Journal 1935-1944: The Fascist Years to

Norman Manea's The Hooligan's Return: A Memoir: the Escape to the

Republic of Letters

De la Jurnalul lui Mihail Sebastian la Întoarcerea huliganului de

Norman Manea: evadarea în republica literelor

Alexandra Vranceanu Pagliardini

\section{(2) OpenEdition}

Journals

Édition électronique

URL : https://journals.openedition.org/ceb/8068

DOI : $10.4000 /$ ceb.8068

ISSN : 2261-4184

Éditeur

INALCO

Édition imprimée

ISBN : 978-2-85831-229-0

ISSN : 0290-7402

Référence électronique

Alexandra Vranceanu Pagliardini, « Du Journal de Mihail Sebastian au Retour du hooligan : une vie de Norman Manea », Cahiers balkaniques [En ligne], 43 | 2015, mis en ligne le 25 juillet 2017, consulté le 06 juillet 2021. URL : http://journals.openedition.org/ceb/8068 ; DOI : https://doi.org/10.4000/ceb.8068

Ce document a été généré automatiquement le 6 juillet 2021.

Cahiers balkaniques est mis à disposition selon les termes de la Licence Creative Commons Attribution - Pas d'Utilisation Commerciale 4.0 International. 


\title{
Du Journal de Mihail Sebastian au Retour du hooligan : une vie de Norman Manea
}

\author{
L'évasion dans la république des lettres \\ From Mihail Sebastian's Journal 1935-1944: The Fascist Years to \\ Norman Manea's The Hooligan's Return: A Memoir: the Escape to the \\ Republic of Letters \\ De la Jurnalul lui Mihail Sebastian la Întoarcerea huliganului de \\ Norman Manea: evadarea în republica literelor
}

Alexandra Vranceanu Pagliardini

\section{Le Journal de Sebastian : le temps de l'écriture et le temps de la lecture}

1 Plus de cinquante ans s'écoulent entre l'écriture du Journal de Mihail Sebastian et sa publication en 1996 en Roumanie. La censure du Parti communiste, qui dominait la vie intellectuelle en Roumanie avant 1989, ne permettait pas une discussion honnête à propos de la position du pays envers les Juifs durant la Seconde Guerre mondiale. Ceci explique le paradoxe que les textes discutés ici aient été publiés à quelques années de distance, alors que leurs écrivains appartiennent à deux mondes différents.

Mihail Sebastian ${ }^{1}$ a vécu entre 1907 et 1945 et il a déployé son activité d'écrivain, de critique littéraire et de journaliste principalement vers la fin des années 1920 et dans les années 1930, une période difficile en Roumanie à cause de la montée au pouvoir des partis fascistes. Sebastian avait eu une grande influence dans la presse littéraire de Bucarest avant d'être marginalisé à la suite de l'institution des lois raciales. Intellectuel raffiné, grand connaisseur des littératures française, anglaise, allemande et russe, il avait été parfois accusé de ne pas être assez Juif par ses coreligionnaires, à cause de ses 
amitiés paradoxales avec des fascistes notoires tel Nae Ionescu, son professeur et directeur du journal Cuvântul, au sein duquel Sebastian était rédacteur. En effet, et son Journal le montre bien, Sebastian a toujours essayé de dépasser les frontières étroites des catégories nationales ou religieuses et de se réfugier dans la république des lettres. Durant la Seconde Guerre mondiale, l'appartenance au monde des intellectuels l'a aidé à survivre et même à faire représenter une de ses pièces, l'Étoile sans nom, au théâtre Alhambra de Bucarest, mais ne lui a pas épargné les nombreuses humiliations de la vie quotidienne. Sebastian est mort en 1945, après l'occupation de la Roumanie par les Soviétiques, dans un accident de voiture.

3 Né en 1936, Norman Manea est un écrivain qui a subi les effets de deux dictatures. À l'âge de cinq ans, il est déporté avec sa famille par le régime fasciste du maréchal Ion Antonescu dans le camp de concentration de Transnistrie, où il perd ses grandsparents; il subit ensuite les limitations imposées aux écrivains par la censure communiste. Après une carrière littéraire impressionnante en Roumanie avec dix livres publiés et plusieurs prix importants, il décide d'émigrer. Son dernier roman publié sous le régime communiste, l'Enveloppe noire, avait tellement souffert à cause des changements imposés par les censeurs du régime de Ceausescu, que Manea en arriva à la conclusion qu'il ne pouvait plus continuer sa carrière d'écrivain en Roumanie ${ }^{2}$. Il vit maintenant aux États-Unis, où il enseigne la littérature européenne au Bard College, et il continue à écrire en roumain. La distance de cinquante ans qui sépare le Journal de Sebastian du Retour du hooligan ${ }^{3}$ de Manea explique les différences entre les deux représentations de la vie en Roumanie aux alentours de la Seconde Guerre mondiale. Dans le Journal de Sebastian l'enregistrement de l'instant vécu est plus important que le commentaire ou l'analyse, et l'histoire semble être le personnage central. Bien évidemment, la recherche du style fait comprendre que Sebastian reste un écrivain même lorsqu'il écrit son journal, mais le fait qu'il ne destine pas son texte à la publication immédiate le pousse à des réflexions parfois très critiques concernant le comportement de ses contemporains et les évènements dramatiques de la Seconde Guerre mondiale. Sa judéité problématique ${ }^{4}$, ses amitiés paradoxales avec des fascistes reconnus, sa culture dans le champ de la littérature et de la musique, ses amitiés dans le monde du théatre et de la diplomatie sont décrites avec finesse et acuité et construisent le portrait d'un homme de lettres qui dépasse les limitations imposées par son identité religieuse à travers l'évasion dans la république des lettres. Sa grande culture et sa connaissance de langues étrangères l'ont aidé jusqu'à un certain point à surpasser les frontières imposées par l'antisémitisme de l'époque et, même lorsqu'il croit avoir raté sa vie, Sebastian ne cesse jamais d'écrire et de lire.

4 Il me semble que c'est à ce niveau que Norman Manea et Mihail Sebastian se rencontrent symboliquement dans leurs textes. Manea reprendra dans le Retour $d u$ hooligan : une vie, la réflexion sur les rapports entre identité nationale, canon littéraire et position sociale ${ }^{5}$. Comme Sebastian auparavant, Manea s'invente un univers construit autour de livres, d'idées, d'amitiés littéraires et un monde de la fiction qui réussit à le protéger du communisme jusqu'au moment de son départ. Dans le chapitre intitulé "Issue de secours", le narrateur raconte sa première expérience de hooligan quand, lorsqu'il avait quatre ans, il a décidé de s'enfuir de chez lui. Sa mère, fâchée, demande au père de le punir, mais « Puis, comme d'habitude, elle prit peur devant la cruauté de la répression, plaida des circonstances atténuantes, la pitié, la grâce du hooligan. Hooligan? Est-ce vraiment le terme qu'elle utilisa?» (Manea, 2003, 172). Après la punition, son père l'a attaché à la table, et Manea interprète symboliquement 
cet évènement comme une prévision de son destin d'écrivain : "Mais entre-temps, il s'était lui-même enchaîné, Ulysse immature, à sa table à écrire » (Manea, 2003, 172).

Dans ce chapitre le narrateur discute plusieurs valeurs du terme «départ» dans le contexte du rapport entre l'écrivain et sa langue. Le départ de Roumanie est vu au début par Manea comme une forme de suicide littéraire, car l'écrivain doit abandonner sa langue : «Je me demandais si l'exil était, pour l'écrivain, l'équivalent du suicide, sans trop d'incertitudes quant à la réponse. Mais la mort qui me guettait ici, à domicile? » (Manea, 2003, 166). L'allusion à la mort fait référence à la censure qui dénaturait ses textes avant de les laisser circuler et c'est ainsi que naîtra la métaphore de la langue comme "maison d'escargot", reprise plusieurs fois par Manea dans ses récits et entretiens.

6 Par rapport à Sebastian, qui ignore cet aspect, Manea donne un rôle central à la langue d'écriture, dans son cas le roumain, qui donne sa véritable identité culturelle à l'écrivain lorsque, exilé, il cherche refuge dans la république des lettres. Mis devant le choix de l'exil, Sebastian l'avait refusé nettement, préférant le risque de rester dans la Roumanie fasciste. Quarante ans plus tard, à cinquante ans, Manea accepte l'exil sans renoncer à être un écrivain roumain, mais en prenant ses distances par rapport à la Roumanie réelle. Ceci explique l'importance du thème de l'identité nationale, religieuse et littéraire dans son œuvre. La relation entre les livres de Manea et de Sebastian est assez évidente, car Manea cite parfois Sebastian, tandis que d'autres fois il fait des références implicites à son Journal.

7 Lorsque le Journal de Sebastian a été publié en 1996, le texte a focalisé l'attention des critiques ${ }^{6}$. Deux raisons principales expliquent cette attention particulière. La première est qu'il s'agit d'un texte convaincant et bien écrit. Avant la publication du Journal, Sebastian était considéré comme un écrivain intéressant, mais plutôt dans un genre mineur. Dans les années 1930, une autre fiction biographique Depuis deux mille ans: roman $^{7}$, dans laquelle il décrit le drame d'un étudiant juif à Bucarest durant le régime fasciste, avait attiré l'attention. Malgré le grand intérêt historique, moral et social du roman Depuis deux mille ans et son écho dans la presse, Sebastian occupait une place marginale dans le canon littéraire et c'est grâce à son Journal qu'il est à nouveau entré dans la conscience du public et des critiques.

La deuxième raison pour laquelle le Journal de Sebastian a suscité l'intérêt des critiques en 1996 est qu'il parle de la fascination pour le fascisme des intellectuels roumains dans les années 1930 et pose le problème du rapport entre culture et politique. Les œuvres de ces intellectuels, tels Eliade et Cioran, mais surtout du philosophe et homme politique Nae Ionescu, avaient été redécouvertes et republiées en Roumanie seulement à partir de 1990, car elles avaient été interdites durant le communisme. Dans les années 1990, les années 1930 étaient vues par les Roumains comme une période de riche activité littéraire et, surtout, une période durant laquelle la littérature roumaine était en contact intense avec la littérature européenne. Après la révolution de 1989, on a examiné en premier lieu les effets de la censure imposée sur la littérature par les communistes $^{8}$, censure qui avait brutalement coupé les liens avec la culture occidentale. Le public découvrait alors, et avec joie, des écrivains roumains parfaitement compatibles avec le monde occidental, comme Eugène Ionesco, Mircea Eliade, Vintilă Horia, Gherasim Luca ou Emil Cioran, ce qui explique pourquoi la littérature des années 1930 jouissait d'un grand prestige. Mais cela explique aussi le 
choc moral devant le Journal de Sebastian, où il décrivait avec vivacité les impressions de certains de ces écrivains au sujet du fascisme.

Après la révolution qui a mis un terme au communisme en Roumanie, les critiques ont opéré un profond changement de canon littéraire et, dans ce processus, le Journal de Sebastian a joué un rôle décisif. Il a mis en question le système des valeurs littéraires en introduisant dans la discussion le rôle moral de l'écrivain et l'importance de ses choix politiques dans des moments historiques difficiles. De ce fait, les intellectuels roumains comprirent l'importance qu'il y avait à mettre en rapport les deux doctrines extrémistes du $\mathrm{xx}^{\mathrm{e}}$ siècle, le fascisme et le communisme, et à discuter de leurs effets sur la culture roumaine.

C'est le mérite de Norman Manea d'avoir mis en relation dans un texte littéraire de grande valeur les similarités, même au niveau de la vie quotidienne, entre ces deux formes d'extrémisme politique. Son roman est une autofiction basée sur le récit de son premier voyage en Roumanie, après dix ans d'exil aux États-Unis, un retour dont le personnage-narrateur a peur et qu'il accepte de faire pour mieux comprendre son rapport avec la Roumanie, qui l'obsède encore.

11 Il y a plusieurs points de contact symbolique entre le récit de Norman Manea et le Journal de Sebastian. Par exemple, l'expérience de la déportation, à laquelle Manea fait référence plusieurs fois, sans jamais la décrire explicitement, apparaît dans le Journal de Sebastian sous la forme d'une obsession constante. À Bucarest, dans l'appartement où il vivait avec sa mère et son frère, Sebastian, angoissé, note dans son Journal: «Le maréchal ${ }^{9}$ aurait pourtant assuré à George Brătianu (selon Rosetti) et au docteur Lupu (selon Braniște) que les natifs juifs n'avaient rien à craindre. Du moins rien de nouveau. Mais je ne puis croire que la modération soit encore possible sur le chemin du pogrom » (Sebastian, Journal, 1998, 393). Sebastian ajoute ensuite une information que nous pouvons lire en la mettant en rapport avec le livre de Manea: «Dimanche, 9 novembre 1941. [...] Un ordre de déportation frappe les Juifs de Dorohoi et de Botoșani » (Sebastian, Journal, 1998, 393) ${ }^{10}$. En effet, il semblerait que, du point de vue littéraire, le texte de Manea continue le Journal de Sebastian et le complète en quelque sorte. Habitant des régions qui ont été frappées par cet ordre, Manea a été déporté à l'âge de cinq ans. Il fait une sorte de description négative, dans le sens où il ne décrit que le retour du camp de Transnistrie ${ }^{11}$ dans un discours elliptique, où il passe de la troisième personne à la première :

En avril 1945, le garçon de neuf ans revenant du camp de Transnistrie découvrait les aliments, les vêtements, l'école, les meubles, les cartes, les jeux: la joie. L'horreur du passé, je l'avais évacuée avec irritation : la "maladie du ghetto »! Je me croyais guéri [...] Puis la chimère de l'écriture m'avait pris sous sa protection. Mais le communisme ne se montrait pas moins antisémite du gouvernement précédent et les haut-parleurs aboyaient, de façon répétée : «étranger, étranger, sans-patrie, anti, impur, anti... ». Je m'étais de nouveau montré, à l'instar de mes ancêtres, indigne de la Patrie.

$\left(\right.$ MANEA $\left.^{12}, 2003,42\right)$

12 On peut difficilement imaginer la vie de l'enfant, privé de tout dans le camp, et aussi son désir de tout oublier, jusqu'à son identité même. À la même époque, à Bucarest, Sebastian enregistre à son tour dans son Journal les humiliations subies par les Juifs durant la guerre : la ration diminuée, l'obligation de payer des taxes au gouvernement, et même des lois qui semblent ridicules dans leur précision et futilité : 
Une ordonnance de la mairie interdit aux Juifs d'aller au marché en dehors d'un certain créneau horaire (dix heures-midi) et prévoit des peines pour les paysans qui vendraient leurs produits aux Juifs en dehors de ces heures. Je me demande chaque jour ce qu'on va encore inventer contre nous. Il faut beaucoup d'imagination pour trouver du nouveau. D'ailleurs, par rapport à l'expropriation des immeubles, aux déportations et aux assassinats, tout le reste devient puéril, grotesque, stupide. Ce n'est même plus déprimant. Il y a parfois dans l'antisémitisme quelque chose de diabolique, mais là, nous ne baignons pas dans le sang, nous pataugeons dans la mesquinerie.

(SEBASTIAN, Journal, 1998, 393-4)

13 L'image donnée par Sebastian au monde "balkanique " de Bucarest, où les lois antisémites étaient suivies par d'autres lois qui permettaient d'éviter le pire, c'est-àdire l'extermination, souligne pourtant la permanente humiliation à laquelle étaient soumis les Juifs. Sebastian commente aussi le caractère ambivalent de l'antisémitisme bucarestois :

Mardi, 5 août 1941. Madeleine Andronescu, au téléphone :

- J'ai honte, Mihail. J'ai honte parce que tu souffres, et pas moi ; j'ai honte parce qu'on t'humilie et pas moi.

C'est quelque chose du même genre que m'a dit Vișoianu (et il n'est pas sentimental, lui), avant-hier je crois, quand un groupe de Juifs est passé à côté de nous :

Chaque fois que je vois un Juif, j'ai envie de m'approcher de lui, de le saluer et de lui dire : « Monsieur, je vous prie de croire que je n'y suis pour rien ».

Le malheur, c'est que personne n'y est pour rien. Tout le monde désapprouve, tout le monde est indigné, mais chacun n'en est pas moins un rouage de cette immense usine antisémite qu'est l'État Roumain (...).

(SEBASTIAN, Journal, 1998, 347)

14 La littérature officielle n'échappe pas à ces lois et Sebastian, qui était à l'époque l'un des intellectuels les plus représentatifs de sa génération se voit exclu à nouveau :

Jeudi, 5 novembre 1942. Aux termes d'un ordre du ministère de la Propagande, les livres des auteurs juifs vont être retirés des librairies et des bibliothèques. J'ai vu aujourd'hui chez Hachette deux immenses tableaux imprimées en gros caractères : « Écrivains juifs ». Mon nom y figure, naturellement, comme celui d'un délinquant, d'un criminel, avec les noms de mes parents, ma date de naissance, la liste de mes livres.

(SEBASTIAN, Journal, 1998, 459)

Il faudrait quand même ajouter que les amis de Sebastian l'ont soutenu durant la guerre et qu'il a réussi à survivre grâce à son activité professionnelle : il a enseigné dans un lycée et dans un collège privé, il a fait des traductions et a même fait représenter une de ses pièces, l'Étoile sans nom, au théâtre Alhambra, sous un autre pseudonyme, le

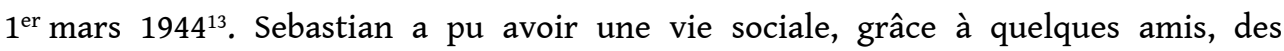
intellectuels :

Lundi, 22 mars 1944. Hier déjeuner à Mogoșoaia avec Rosetti, Camil, un prince italien, un religieux français et un diplomate suisse. Marthe Bibesco plus simple, moins somptueuse qu'autrefois.

(SEBASTIAN, Journal, 1998, 485)

Mais il s'agit tout de même d'une existence de marginal et Sebastian sent qu'il a raté sa vie. Dans un passage du 27 mai 1942 où il parle du destin de son ami de jeunesse, Mircea Eliade ${ }^{14}$, qui se trouve à Lisbonne depuis le début de la guerre, il commente sa marginalisation : 
Tandis qu'il mène une existence de magnat, dans des régions paradisiaques, une vie de paix et de luxe, de confort de rêve, tandis qu'il profite de "l'ordre nouveau ", moi, ici, je traîne une misérable vie de prisonnier. Et après la guerre, à supposer que j'en réchappe et que nous nous revoyions, je ne pourrai opposer à ses années d'épanouissement que mes tristes années d'humiliation, de ratage. Rien n'excuse jamais le ratage. Les victoires, même si elles sont le fruit d'une infamie morale, restent des victoires.

(SEBASTIAN, Journal, 1998, 432)

Sebastian avait, un an plus tôt, enregistré son exclusion du canon de la littérature nationale, opérée par le critique littéraire George Călinescu :

Jeudi, 14 juillet 1941. J'ai lu hier [...] la page que me consacre Călinescu dans son Histoire de la littérature [roumaine]. C'est probablement ce qui a été écrit de plus sévère à mon égard. Pas de talent artistique, pas d'aptitude à l'écriture. Il commence par le premier jugement et finit par le second. Cela m'a agacé sans plus. Désagréable, cette page dans une histoire de la littérature dont le caractère monumental en fait une référence incontournable. Un livre pareil - mille pages in quarto - parait tous les trente ou quarante ans. Il faudra donc attendre une quarantaine d'années pour avoir un autre point de vue.

(SEBASTIAN, Journal, 1998, 338)

18 En effet, il faudra attendre la publication du Journal pour assister à un véritable changement et pour commencer à discuter les limites du canon littéraire conçu autour des valeurs liées à l'identité nationale ${ }^{15}$.

\section{Sebastian et Manea, deux « hooligans » de la littérature}

19 Pourquoi donc Norman Manea choisit-il comme modèle intellectuel Mihail Sebastian? Une clé de lecture vient du terme "hooligan », qui apparaît dans le titre du roman de Manea. Le mot hooligan avait un sens particulier en roumain dans les années 1930, il désignait alors les jeunes intellectuels groupés autour du professeur Nae Ionescu, le directeur du journal à tendance fasciste Cuvântul [La parole]. Bien que Juif, Sebastian était un ami et collaborateur de Nae Ionescu, qui l'appréciait beaucoup sur le plan intellectuel, professionnel et affectif. En effet, Sebastian était, jusqu'à l'interdiction de la revue Cuvântul et l'emprisonnement de Nae Ionescu qui mourra ensuite dans des conditions mystérieuses, un ami très proche de plusieurs intellectuels roumains fascistes, appelés des « légionnaires ».

L'amitié paradoxale entre le Juif Sebastian et l'homme politique fasciste Nae Ionescu ${ }^{16}$ est la source biographique d'un roman très original, Depuis deux mille ans, où l'auteur décrit la grande amitié entre maître et disciple, en faisant référence à sa relation avec Nae Ionescu. Du point de vue du genre littéraire, Depuis deux mille ans. Roman se construit sur une formule à mi-chemin entre le roman de formation et l'autofiction. Le texte, d'une grande originalité littéraire, apparaît en 1934 accompagné d'une préface, violemment antisémite, signée par l'objet de l'adoration respectueuse de Sebastian, c'est-à-dire Nae Ionescu. Les critiques des années 1930 l'ont lu comme un roman à clef et le scandale littéraire qui a suivi sa parution place Sebastian dans une situation particulière: d'une part, il est considéré par ses coreligionnaires comme un " hooligan ", un ami des fascistes ; d'autre part, il reste quand même un Juif pour ses amis roumains, c'est-à-dire un marginal et un étranger. Sebastian expliquera sa situation paradoxale et tragique dans un autre livre, intitulé Cum am devenit huligan 
[Comment je suis devenu hooligan], publié en 1935, où il commente avec finesse le dossier de presse du roman Depuis deux mille ans. C'est peut-être ici que se trouve la clef de lecture pour le Retour du hooligan de Manea.

Comment et pourquoi Manea voulait-il prendre le nom de « hooligan $»^{17}$ ? En quoi est-il semblable à Mihail Sebastian? J'avance une hypothèse de lecture. Avec son roman, dans lequel il décrit le drame d'un étudiant juif à Bucarest entre 1930 et 1933, Sebastian osait adopter un sujet difficile - la tragédie d'un homme de culture qui cherche à s'intégrer dans la littérature nationale roumaine et qui ne réussit qu'à se faire exclure par tout le monde. En choisissant de publier la préface de son maitre, Nae Ionescu, qui écrit que l'auteur du livre ne sera jamais " un Roumain de Brăila », mais restera " un Juif de Brăila", Sebastian ose encore plus, car il montre les limites profondes d'un système culturel construit autour du « spécifiquement national ».

Si nous analysons l'intertexte formé, d'une part, par Depuis deux mille ans, la préface de Nae Ionescu et l'essai polémique de Sebastian Comment je suis devenu hooligan, auquel s'ajoutent, d'autre part, en 1996 le Journal, puis, en 2006, l'autofiction de Manea, le tableau commence à devenir complet et le terme hooligan montre sa signification symbolique. C'est dans ce sens que Manea adopte le terme de « hooligan » et non dans le sens donné à ce mot par Mircea Eliade dans son roman Huliganii de 1935, où Eliade l'utilise pour désigner les jeunes intellectuels qui veulent détruire ce qui est « vieux »: «Il y a un seul début fertile dans la vie : l'expérience hooliganique » (Eliade, 1987, 200).

Par le choix de ce mot dans le titre de son ouvrage, Manea souligne sa position intermédiaire: comme écrivain juif, il se sent exclu du canon littéraire roumain; comme écrivain roumain qui habite à New York et qui continue à écrire en roumain, il reste en dehors des hiérarchies nationales ${ }^{18}$. Dans le chapitre intitulé la Maison de l'escargot, Manea raconte son rapport difficile avec « la maison », avec les maisons qu'il a dû abandonner tour à tour et montre comment il a trouvé « refuge dans la maison que seul le Livre me promettait» (Manea, 2003, 261) ${ }^{19}$. D'ailleurs, en pressentant son destin de futur exilé, lorsqu'il avait publié en 1969 son premier livre, Manea avait pensé :

J'avais enfin trouvé mon domicile véritable. La langue permet non seulement la résurrection, mais aussi la légitimation, la citoyenneté, l'appartenance authentique. Se voir chassé y compris de cet ultime refuge constitue l'exil le plus violent, un incendie qui atteint le Cœur même de l'être.

(MANEA, 2003, 261-262)

La censure communiste, l'antisémitisme du régime de Ceausescu l'avaient chassé vers l'Occident ; cela n'empêchera pas Manea de garder son identité roumaine ni de la faire cohabiter avec ses autres identités juive ${ }^{20}$ et américaine :

Il ne me restait plus qu'à emporter ma langue avec moi, comme une maison. La maison de l'escargot. Sur quelque ravage que j'échoue, elle serait pour moi, je le savais, le refuge infantile de la survie.

(MANEA, 2003, 262)

Manea reprend le personnage intellectuel du hooligan comme un symbole de l'attaque contre les structures nationalistes de la littérature. Sebastian avait déjà posé ce problème dans son roman de 1934, Depuis deux mille ans, mais à l'époque, sa voix ne pouvait être entendue :

Je voudrais connaître, par exemple, la législation antisémite qui pourrait abolir en moi ce fait irrévocable : je suis né au bord du Danube, dans une contrée que j'aime.

(SEBASTIAN, Depuis deux mille ans, 1998, 330) 
26

Pendant la Seconde Guerre mondiale, la position ambiguë et l'identité hybride de Sebastian avaient seulement provoqué la rage des antisémites et le désarroi des Juifs roumains; dans la Roumanie communiste son texte ne pouvait pas être publié sans subir une censure déformante. Mais le texte de Manea a eu la chance d'être publié à un autre moment. Il reprend et réinvente dans le récit des évènements dont il a été témoin un personnage à identité hybride, mais qui, cette fois, au lieu d'être un exclu, devient le symbole du nouveau monde globalisé : l'écrivain migrant.

\section{Deux autobiographies spirituelles : échapper à tous les nationalismes grâce à la littérature}

J'arrive ainsi à expliquer le titre de mon article. J'interprète le Retour du hooligan comme le retour de l'écrivain migrant qui a dépassé les frontières de la littérature nationale et qui a trouvé une place dans la république des lettres, une république mondialisée, qui accepte que l'écrivain porte avec lui sa maison d'escargot - c'est-à-dire sa langue et sa culture ou ses cultures d'origine. La métaphore de la Maison d'escargot désigne chez Manea la langue roumaine, mais peut-on considérer Manea comme un écrivain roumain tout court? Se considère-t-il ainsi ? On peut sentir une certaine amertume dans ses propos lorsqu'il parle de son pays d'origine, ce qui rappelle sans doute Sebastian.

Il y a des ressemblances entre le destin de l'œuvre de Manea et celle de Sebastian au niveau de la circulation et de la traduction. Grâce au fait que leurs écrits ont été traduits dans des langues de large diffusion, le Journal et le Retour du hooligan ont pu bénéficier d'un public multinational. Cette circulation au-delà des frontières nationales change beaucoup la perspective de lecture parce qu'elle permet de juger le texte pour sa valeur littéraire ou éthique. La traduction et la circulation ont permis à Manea et à Sebastian l'entrée dans la république des lettres, telle qu'elle est devenue à l'époque de la globalisation.

Formellement, le Journal de Sebastian et le Retour du hooligan se ressemblent beaucoup : deux autobiographies intellectuelles écrites par des écrivains d'origine juive, qui habitent dans des univers concentrationnaires - sous le fascisme ou sous le communisme - et qui cherchent à s'évader grâce à la république des lettres. La confession et la recherche de soi occupent la place centrale dans ces deux textes - et cette opération se fait grâce à l'interprétation de l'histoire. Les notations concernant l'évasion de Sebastian dans le monde de l'écriture et de la lecture sont trop nombreuses pour être citées en détail : il traduit Shakespeare et Jane Austen, il lit Balzac, Gide et Proust, il écrit deux pièces de théâtre et un roman entre 1940 et 1945 . Il allait à l'Université de Bucarest donner son premier cours de littérature universelle lorsqu'il a été accidenté mortellement par un camion soviétique. Manea aussi écrit un texte plein de références intertextuelles. Se chercher dans d'autres textes, se trouver grâce à la littérature reste, me semble-t-il, une caractéristique fondamentale de la vie pendant la Seconde Guerre mondiale. Souvenons-nous que Erich Auerbach, exilé à Istanbul durant cette même guerre cherchait refuge dans ses recherches sur la circulation d'un concept, la mimesis, à travers les âges et les cultures, dans la littérature européenne. Toujours durant la guerre, à l'autre bout de l'Europe, Ernst Robert Curtius voyait l'unité de la littérature européenne dans les topoï qui avaient circulé depuis l'Antiquité, durant 
le Moyen Âge et jusqu'à la période moderne. Il me semble que cette évasion des intellectuels dans une recherche, utopique peut-être, d'une république des lettres pouvant offrir refuge dans des moments de crise est aussi pratiquée par Sebastian et Manea.

\section{BIBLIOGRAPHIE}

CĂLINESCU Matei, 1987, “On Norman Manea's The Hooligan's Return”, in Alvin H. ROSENFELD (ed.), The Writer Uprooted: Contemporary Jewish Exile Literature, Bloomigton: Indiana University Press, pp. 27-51.

ELIADE Mircea, 1987 [1935], les Hooligans, Paris : L'Herne, trad. fr. Alain Paruit.

LIVEZEANU Irina, 2004, « les guerres culturelles en Roumanie post-communiste : débat intellectuels sur le passé récent », in Catherine DURANDIN, Magda CÂRNECI (dir.), Perspectives roumaines : du postcommunisme à l'intégration européenne, Paris : L'Harmattan.

MANEA Norman, 2003, le Retour du hooligan : une vie, Paris : Seuil, trad. fr. Nicolas Véron et Odile Serre.

MANEA Norman, 2009, l'Enveloppe noire, Paris : Seuil, trad. fr. Marily Le Nir.

MANEA Norman, «Puloverul», in Variante la un autoportret, Iași : Polirom, p. 13-29.

MÂNDRA Mihai, 2007, "Identity and Alterity: Geographies of the Mind", in University of Bucharest Review. A Journal of Literary and Cultural Studies, vol. IX, 1/2007, pp. 129-135.

OIȘTEANU Andrei, 2011, "Mihail Sebastian and Mircea Eliade: Chronicle of a Broken Friendship", in Valentina GLAJAR, Jeanine TEODORESCU (eds), Local History, Transnational Memory in the Romanian Holocaust, New York: Palgrave Macmillan, pp. 119-137.

PETREU Marta, 2009, Diavolul și ucenicul său: Nae Ionescu et Mihail Sebastian [Le Diable et son apprenti : Nae Ionescu et Mihail Sebastian], Iași : Polirom.

POLOUEKTOVA Ksenia, 2009, “'Is There a Place like Home?' Jewish Narratives of Exile and Homecoming in Late Twentieth-Century East-Central Europe”, in John NEUBAUER, Borbala Zsuzsanna TÖRÖK (eds), The Exile and Return of Writers from East-Central Europe: A Compendium, Berlin, New York: Walter de Gruyter, pp. 424-473.

SEBASTIAN Mihail, 1998a [1996], Journal : 1935-1944, Paris : Stock, trad. fr. Alain Paruit. SEBASTIAN Mihail, 1998b [1934], Depuis deux mille ans : roman, Paris : Stock, trad. fr. Alain Paruit. SIMUȚ Ion, 2008, «AmbiguităŢile exilului», in România literară, 15/2008. http://www.romlit.ro/ambiguitile_exilului [consulté le $1^{\mathrm{er}}$ février 2016]

\section{NOTES}

1. Son nom était Josif Hechter, Mihail Sebastian son pseudonyme littéraire. 
2. Norman MANEA a ensuite réécrit son roman, qui est publié aussi en traduction française : 2009, l'Enveloppe noire, trad. Marily Le Nir.

3. Le Retour du hooligan a été écrit en roumain, publié en 2003 en traduction anglaise et en 2006 en roumain et en traduction française (Paris : Seuil), a reçu de nombreux prix, dont le Médicis Étranger en 2006.

4. Voir Marta PETREU, Diavolul și ucenicul său: Nae Ionescu et Mihail Sebastian, 2009.

5. Lorsque Manea revient en Roumanie, en 1996, le Journal de Sebastian est au centre de l'attention et cela explique peut-être la puissante relation intertextuelle qui existe entre ces deux textes.

6. Sur les réactions à cet évènement éditorial, voir Irina LIVEZEANU, 2004, « les guerres culturelles en Roumanie post-communiste: débat intellectuels sur le passé récent ", surtout les pages 22-30.

7. Traduction française publiée à Paris : Stock, 1998.

8. Après l'occupation de la Roumanie par les Soviétiques en 1945, la culture roumaine a été décapitée: tous les intellectuels qui n'ont pas accepté de falsifier l'histoire des Roumains en soulignant la «grande amitié entre les Russes et les Roumains » ont été interdits et souvent emprisonnés. L'Académie Roumaine a été remplie par des sympathisants des Soviétiques. Les écrivains exilés, comme Eliade, Ionesco, Vintilă Horia, etc., ont été interdits - absents des bibliothèques et non republiés, leurs livres brûlés. Voilà pourquoi les écrivains roumains des années 1930-1940, victimes du communisme, ont été idéalisés. Le fait que le Journal de Sebastian soit publié pour la première fois en même temps que les romans d'Eliade a généré une certaine compétition entre les deux : le premier, Juif, le deuxième, sympathisant du mouvement fasciste des légionnaires roumains. La vie durant la Seconde Guerre mondiale ne peut être récupérée en ce moment, du point de vue littéraire, qu'à travers cette rupture énorme de 50 ans, et ainsi fait que de nombreuses œuvres écrites alors ont été lues beaucoup plus tard.

9. Il s'agit du maréchal Ion Antonescu, chef de l'État roumain durant la Seconde Guerre mondiale.

10. Avant l'introduction des lois antisémites, Sebastian faisait partie des jeunes intellectuels les plus prometteurs et les plus influents : comme rédacteur à Cuvântul, un journal très lu à l'époque, il avait la possibilité d'exprimer son opinion sur toutes les publications littéraires - il le faisait souvent et s'est fait de nombreux ennemis littéraires à cause de ses critiques acides. Mais, après 1941, Sebastian subit une marginalisation constante.

11. Dans ses nouvelles, il reprend des moments vécus dans le camp de concentration de Transnistrie, mais la nouvelle Puloverul, republiée dans Manea 2008, est un texte moins autofictionnel que le Retour du hooligan.

12. MANEA, 2003, le Retour du hooligan : une vie, trad. fr. Nicolas Véron et Odile Serre.

13. Le mardi 14 novembre, il raconte son entretien avec Soare, le metteur en scène, qui lui dit : «C'est un chef d'œuvre » (SEBASTIAN, Journal, 1998, p. 513).

14. Voir aussi Andrei OIșTEANU, 2011, "Mihail Sebastian and Mircea Eliade: Chronicle of a Broken Friendship".

15. À son tour, Manea reprend les mêmes problèmes. Il se sent aussi exclu de la littérature roumaine, marginalisé et le thème est récurent dans le Retour du hooligan. 
16. Voir PETREU, 2009.

17. Voir aussi Mihai MÂNDRA, 2007, "Identity and Alterity: Geographies of the Mind".

18. Voir pour le thème du retour chez les écrivains exilés ou migrants, POLOUEKTOVA, 2009, “'Is There a Place like Home?' Jewish Narratives of Exile and Homecoming in Late Twentieth-Century East-Central Europe”, 424-473, et concernant le Retour du hooligan, POLOUEKTOVA, 2009, 462-467.

19. «Seul dans cette chambre, étrangère et sombre, de la maison Riemer de Fălticeni, et seul dans l'univers, je découvrais tout à coup une autre maison, un autre monde, un autre moi-même. Les livres donneraient asile, par la suite, à mes années d'apprentissage, à Suceava, à Bucarest, dans tous les refuges où j'emporterais ma seule richesse, ma valise d'illusions. [...] Je continuais de chercher refuge dans la maison que seul le Livre me promettait. Exil, maladie salvatrice? Un va-et-vient permanent entre moi et mon double : dans la tentative de me trouver, de me substituer à moi-même, de me perdre pour tout commencer depuis le début. [...]» (MANEA, 2003, 261).

20. Voir Ion SIMUȚ, 2008, «AmbiguităȚile exilului».

\section{RÉSUMÉS}

J'analyserai ici l'image de la Seconde Guerre mondiale en Roumanie telle qu'elle apparaît dans l'intertexte généré par le roman autofictionnel le Retour du hooligan : une vie de Norman Manea et par le Journal : 1935-1944 écrit par Mihail Sebastian. Le Journal de Sebastian raconte la période de la Seconde Guerre mondiale, mais le texte a été publié en roumain seulement en 1996 (la traduction française a été publiée à Paris : Stock, 2007). Ces deux écrivains roumains ont subi l'expérience de la marginalisation (Sebastian) et de la déportation (Manea) parce qu'ils étaient Juifs, et ils l'ont racontée dans leurs écrits. Dans son autofiction le Retour du hooligan: une vie, Manea fait de nombreuses références à la Seconde Guerre mondiale en Roumanie, même si l'action principale du texte a lieu à New York et à Bucarest en 1998. L'expérience traumatique subie par Sebastian durant la guerre est revisitée et interprétée par Manea dans un jeu de miroirs autofictionnel ; je m'occuperai des moyens par lesquels l'écriture du moi se transforme en témoin de l'histoire.

I'll analyse in this article the representation of the Second World War in Romania as seen in the inter-text generated by Norman Manea's autofiction The Hooligan's Return: A Memoir and from Mihail Sebastian's Journal 1935-1944: The Fascist Years. In The Hooligan's Return, Manea describes scenes from the Second World War in Romania, even if the main action takes place in New York and Bucharest in 1998. The traumatic experience Mihail Sebastian suffered during the war is revisited and interpreted by Norman Manea in an autofictional mirror game; I'll analyse the way in which autobiographical writing becomes the witness of history.

Voi analiza aici imaginea celui de-al doilea război mondial în România așa cum apare ea din intertextul generat de romanul autoficțional Întoarcerea huliganului de Norman Manea și din Jurnal: 1935-1944 scris de Mihail Sebastian. În Întoarcerea huliganului, Norman Manea face numeroase trimiteri la cel de-al doilea război mondial în România, chiar dacă acțiunea principală a textului se petrece la New York și la București în1998. ExperienȚa traumatică suferită 
Mihail Sebastian în timpul războiului este revizitată și înterpretată de Norman Manea într-un joc de oglinzi autoficțional; mă voi ocupa de felul în care scriitura autobiografică devine martor al istoriei.

\section{INDEX}

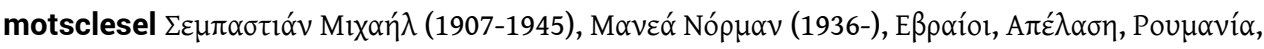

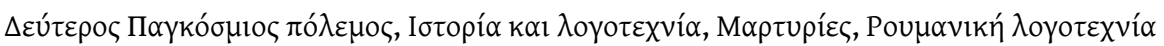
motsclestr Sebastian Mihail (1907-1945), Manea Norman (1936-), Yahudi, Sürgün, Romanya, İkinci Dünya Savaşı, Tarihi ve Edebiyatı, Tanıklar, Romen edebiyatı motsclesmk СЕБАСТЬЯН МИХАИЛ (1907-1945), МАНЯ НОРМАН (1936), ЕВРЕИТЕ, ДЕПОРТАЦИЈА, РОМАНИЈА, ВТОРАТА СВЕТСКА ВОЈНА, ИСТОРИЈА И ЛИТЕРАТУРА, СВЕДОШТВА, РОМАНСКА КНИЖЕВНОСТ

Keywords : Sebastian Mihail (1907-1945), Manea Norman (1936-), Jews, Deportation, Rumania, Second World War, History and literature, Testimonies, Rumanian literature

Mots-clés : Sebastian Mihail (1907-1945), Sebastian Mihail (1907-1945), Manea Norman (1936-), Manea Norman (1936-), juif, juif, déportation, déportation

Thèmes : Histoire, Littérature roumaine

Index géographique : Roumanie

Index chronologique : guerre mondiale (1939-1945)

\section{AUTEUR}

\section{ALEXANDRA VRANCEANU PAGLIARDINI}

Université de Bucarest 